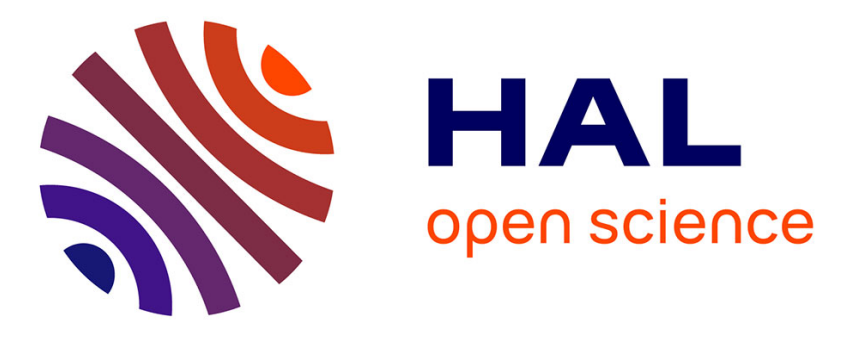

\title{
Bose soft disks: a minimal model for supersolidity
}

Sebastiano Saccani, Saverio Moroni, Ettore Vitali, Massimo Boninsegni

\section{To cite this version:}

Sebastiano Saccani, Saverio Moroni, Ettore Vitali, Massimo Boninsegni. Bose soft disks: a minimal model for supersolidity. Molecular Physics, 2012, 109 (23-24), pp.2807-2812. 10.1080/00268976.2011.615346 . hal-00771583

\section{HAL Id: hal-00771583 https://hal.science/hal-00771583}

Submitted on 9 Jan 2013

HAL is a multi-disciplinary open access archive for the deposit and dissemination of scientific research documents, whether they are published or not. The documents may come from teaching and research institutions in France or abroad, or from public or private research centers.
L'archive ouverte pluridisciplinaire HAL, est destinée au dépôt et à la diffusion de documents scientifiques de niveau recherche, publiés ou non, émanant des établissements d'enseignement et de recherche français ou étrangers, des laboratoires publics ou privés. 


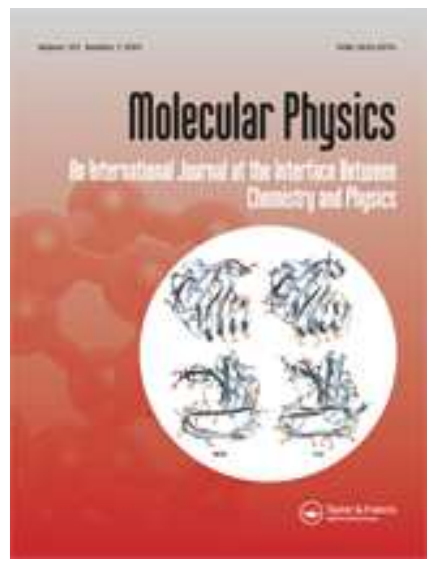

\section{Bose soft disks: a minimal model for supersolidity}

\begin{tabular}{|r|l|}
\hline Journal: & Molecular Physics \\
\hline Manuscript ID: & TMPH-2011-0208.R1 \\
\hline Manuscript Type: & Special Issue in honour of Luciano Reatto \\
\hline Author: & 25 -Jul-2011 \\
\hline Complete List of Authors: & $\begin{array}{l}\text { Saccani, Sebastiano; SISSA } \\
\text { Moroni, Saverio; IOM-CNR } \\
\text { Vitali, Ettore; Universita' degli studi di Milano } \\
\text { Boninsegni, Massimo; University of Alberta }\end{array}$ \\
\hline Keywords: & Supersolid, Cluster Solid, Bose Gas \\
\hline $\begin{array}{l}\text { Note: The following files were submitted by the author for peer review, but cannot be converted } \\
\text { to PDF. You must view these files (e.g. movies) online. }\end{array}$ \\
\hline $\begin{array}{l}\text { p_R1.tex } \\
\text { files.zip }\end{array}$ \\
\hline
\end{tabular}

\section{SCHOLARONE Manuscripts}


Molecular Physics

Vol. 00, No. 00, Month 200x, 1-8

\title{
RESEARCH ARTICLE
}

\section{Bose soft disks: a minimal model for supersolidity}

\author{
S. Saccani ${ }^{1}$, S. Moroni ${ }^{1}$, E. Vitali ${ }^{2}$, and M. Boninsegni ${ }^{3}$ \\ ${ }^{1}$ SISSA Scuola Internazionale Superiore di Studi Avanzati and DEMOCRITOS National \\ Simulation Center, Istituto Officina dei Materiali del CNR Via Bonomea 265, I-34136, \\ Trieste, Italy \\ ${ }^{2}$ Dipartimento di Fisica, Università degli Studi di Milano, via Celoria 16, 20133 Milano, \\ Italy \\ ${ }^{3}$ Department of Physics, University of Alberta, Edmonton, Alberta, Canada T6G 2G7 \\ (Received 00 Month 200x; final version received 00 Month 200x)
}

\begin{abstract}
The low temperature phase diagram of an assembly of Bose particles, interacting via a simple soft disk potential, is studied numerically by means of Monte Carlo simulations. At sufficiently high densities, the system features a cluster crystal phase, as observed in its classical counterpart. In the low temperature limit, and in a relatively wide region of parameter space, the quantum cluster crystal undergoes a transition to a supersolid phase, displaying both a finite superfluid fraction and crystalline order. We argue that soft-core Bosons ought be regarded as a "minimal model" of continuous-space supersolidity. With the aim of elucidating the interplay between superfluid response and solid order, we compute the excitation spectrum of the system. A comparison of that of the insulating cluster crystal with that of the supersolid, shows that the latter features an additional acoustic mode.
\end{abstract}

Keywords: Supersolidity, Cluster Solid, Bose Gas

\section{Introduction}

The elusive supersolid phase of matter has intrigued condensed matter and manybody physicists for over half a century. The observation in 2004 by Kim and Chan [1] of non-classical rotational inertia in a crystal of ${ }^{4} \mathrm{He}$ rekindled the interest in such a phase of experimenters and theorists alike. Solid ${ }^{4} \mathrm{He}$ is universally believed to be the most likely candidate to display a supersolid phase, among naturally occurring condensed matter systems. At the time of this writing, however, the controversy over whether the anomaly observed by Kim and Chan indeed signals the transition of the system to a supersolid phase, has not yet been resolved [2]. Besides the feebleness of the observed superfluid response, as well its puzzling dependence on details of the measuring apparatus, it seems as if a significant source of ambiguity in the interpretation of existing data stems from the currently generally limited understanding of the supersolid phase.

In order to achieve greater insight, it would be beneficial to identify a theoretical many-body system whose supersolid properties could be established unambiguously, by means of rigorous calculations. That would allow one to characterize the phase not only through its most obvious qualities (namely superflow and crystalline order), but also some, like dynamical responses, that could be probed by different experiments than those carried out until now. Presumably, that would not only 
help resolve the controversy over solid helium, but also facilitate the detection of supersolid phases in other systems.

Recently, Cinti et al. [3] investigated by Monte Carlo simulations a twodimensional model of bosons interacting via a purely repulsive pair-wise potential, featuring a dipolar $1 / r^{3}$ long-range tail but saturating to a constant finite value at short distance. This particular form of potential might be engineered in cold atomic systems, through a mechanism known as Rydberg blockade [4]. Such a system displays for sufficiently high density a crystal phase, i.e., one in which each lattice site is occupied by a number of particles $K$ larger than one. In turn, such a crystal can turn superfluid at low enough temperature, phase coherence being established by quantum-mechanical tunnelling of particles across adjacent lattice sites.

Bounded pair potentials have been employed in classical systems as well, to describe the effective interactions between macromolecules. In that context too, it was found that a certain class of bounded repulsive pair potentials give rise, for sufficiently high density and low temperature, to multiple-occupancy crystal phases [5]. In particular, a criterion is known for the quantitative prediction of clustering, based on the form of the inter-particle potential [6]. We refer henceforth to the bunch of particles at each lattice site, as "cluster" or "droplet", and to the resulting phase as "cluster crystal" ("cluster supersolid" when appropriate).

The possible relevance of the long-range tail of the interaction, in stabilizing the supersolid phase, was recently studied [7] by considering a strictly short-range, soft disk type inter-particle potential,

$$
v(r)= \begin{cases}D & \text { if } r \leq a \\ 0 & \text { if } r>a\end{cases}
$$

where $D$ is the potential barrier that two particles have to overcome in order to overlap (independent of the extent of the overlap), and $a$ is the disk radius. The similarity between the phase diagram obtained with this simple interaction [7] and that found in the presence of a dipolar (or faster-decaying) tail [3], points to the fundamental irrelevance of the long-range tail of the potential. The soft-disk system can thus be considered a "minimal" underlying model for the presence of supersolidity in the continuum. As such, it is a worthwhile subject for further investigation for the reasons mentioned above, namely as a toy model embodying basic properties of the supersolid phase.

With this aim, in this work we carry out a numerical study of several key properties of the soft disk model and its supersolid phase. Specifically, we compute the momentum distribution, the potential felt by a test particle (related to tunnelling of particles between different clusters), and the superfluid fraction. We also explore the evolution of the statistics of cycles of permutation of identical particles across the supersolid transition, and compare the excitation spectra of the supersolid and the insulating crystals.

The paper is organized as follows: in the next section we introduce the notation and briefly describe the computational methodology, devoting the rest of the manuscript to the presentation of the results. 


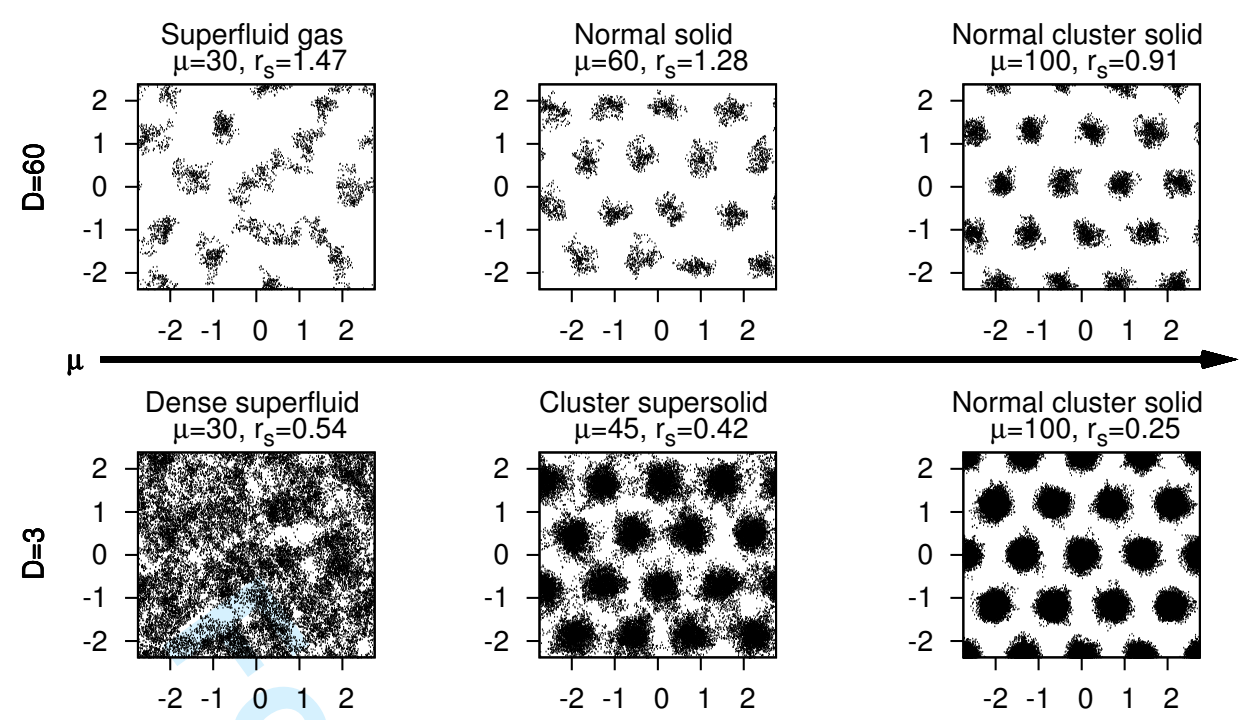

Figure 1. Qualitative low temperature phase diagram for high and low $D$ as a function of $\mu$. The panels show typical spatial configuration of the world lines resulting from simulations at low $T$, referring to the various phases. Results shown in the upper and the lower part of the figure correspond to simulations with $D=60$ and $D=3$, respectively.

\section{Model and methodology}

We consider a system of Bose particles of spin zero in two dimensions. The Hamiltonian of the system is

$$
\mathcal{H}=-\frac{1}{2} \sum_{i=1}^{N} \nabla_{i}^{2}+D \sum_{i>j} \Theta\left(1-r_{i j}\right)
$$

where $r_{i j}$ is the distance between particles $i$ and $j$, all lengths are expressed in units of the soft-core diameter $a$, while all energies are expressed in units of $\epsilon_{\circ}=\hbar^{2} / m a^{2}$. The parameter $D \equiv V / \epsilon_{\circ}$ can also be expressed as $(a / \xi)^{2}$, where $\xi$ is the quantummechanical penetration length of a potential barrier of height $V$. In the limit $\xi \rightarrow 0$ the model (2) reduces to the hard-sphere gas.

Path Integral Monte Carlo simulations of the system described by (2), enclosed in a cell with periodic boundary conditions of sides $\left(L_{x}, L_{y}\right)$, have been performed by means of the Continuous-space Worm Algorithm in the grand canonical ensemble (i.e. at fixed temperature $T$, area $A$ and chemical potential $\mu$ ). Because this methodology is by now well-established, and is thoroughly described elsewhere, we omit here technical details, and refer the interested reader to the original references $[8,9]$. We denote by $N$ the average number of particle, by $N_{c}$ the number of clusters contained in the simulation cell and by $l$ the lattice parameter. We express the density $\rho$ in terms of the dimensionless parameter $r_{s}=1 / \sqrt{\rho a^{2}}$. The presence of a global superfluid response, extending to the whole system, is assessed numerically through the winding number estimator [10] of the superfluid fraction $\rho_{s}$.

\section{Results}

In Figure 1 we summarize the main features of the phase diagram obtained in Ref. [7]. In the $T=0$ limit, it is possible to identify two qualitatively different behaviors of the system in the strongly interacting (high $D$ ) and weakly interacting (low $D$ ) regimes. In the former case $(D=60$ in Figure 1$)$, for low values of the density the 


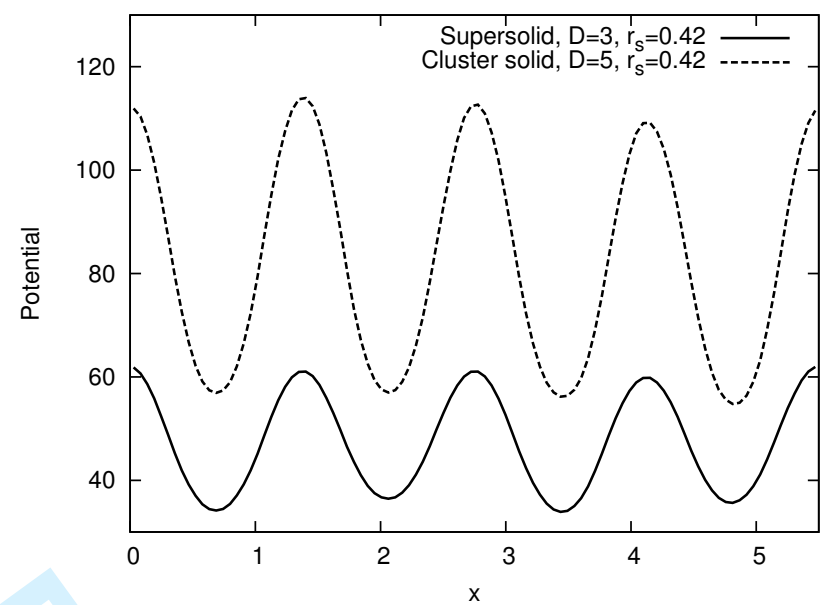

Figure 2. Potential felt by a test particle averaged over many configurations along the cristallographic direction $[1,0]$ passing trough the lattice sites, for two different heights of the interparticle potential. The minima of the potential correspond to the positions of the clusters, where the disk density is higher. The barrier between neighbouring lattice sites is increased for higher values of $D$, progressively supressing tunnelling probability.

system is a superfluid gas, essentially behaving like an hard-disk fluid. This phase undergoes solidification into a triangular crystal on increasing $\mu$. For sufficiently large values of $D$, the number of particles per cluster (unit cell) is $K=1$. However, on further increasing the chemical potential, particles bunch into clusters which organize in a solid preserving the triangular structure. This phenomenon is classical in nature, being related to the energy cost associated to particles being at a distance less than the soft core diameter.

For lower values of $D$, the system can be kept superfluid up to a much higher density $\left(r_{s}<1\right.$, meaning that particles easily overlap). Above a certain $\mu$ the system crystallizes directly into a cluster crystal with $K>1$, the exact value depending on $D$. Moreover, even though density modulations appear in the system, the superfluid fraction in the system is not fully suppressed. This is the aforementioned cluster supersolid phase. This phenomenon is underlain by long cycles of exchanges of particles permutations between neighbouring clusters, eventually leading to the superfluidity of the system as a whole.

Upon further increase of the density, $\rho_{s}$ is progressively suppressed by the rising potential barrier between neighbouring lattice sites, which is associated with tunnelling probability. This eventually destroys phase coherence in the system, which goes into a normal (i.e. non superfluid) cluster phase. The same effect of tunnelling suppression can be obtained also by increasing $D$ at constant $r_{s}$, instead of $\mu$, as can be seen in Figure 2, where the averaged potential felt by a "test" particle is plotted along the cristallographic direction $[1,0]$ passing trough the lattice sites. Note that the potential has minima in correspondence of the clusters. Higher potential barriers lead to increasingly compact clusters, i.e. particles pile up on a smaller spatial region (compare, e.g., the spread of the world lines in the two right panels of Figure 1). The pattern illustrated in Figure 2 suggests a correpondence with a soft-core Hubbard model, although in the present case the triangular lattice is self-assembled and not superimposed.

Finally, contraction of the world lines with ensuing demotion of tunnelling occurs when the temperature is increased. This is reflected in the distribution of the lengths of permutation cycles, shown in Figure 3, main panel. At low temperatures long permutation cycles can take place, involving particles pertaining to different clusters. At higher temperatures permutation cycles longer than the number of particles $K$ inside a single droplet, i.e. longer than 6 or 7 in Figure 3, are 


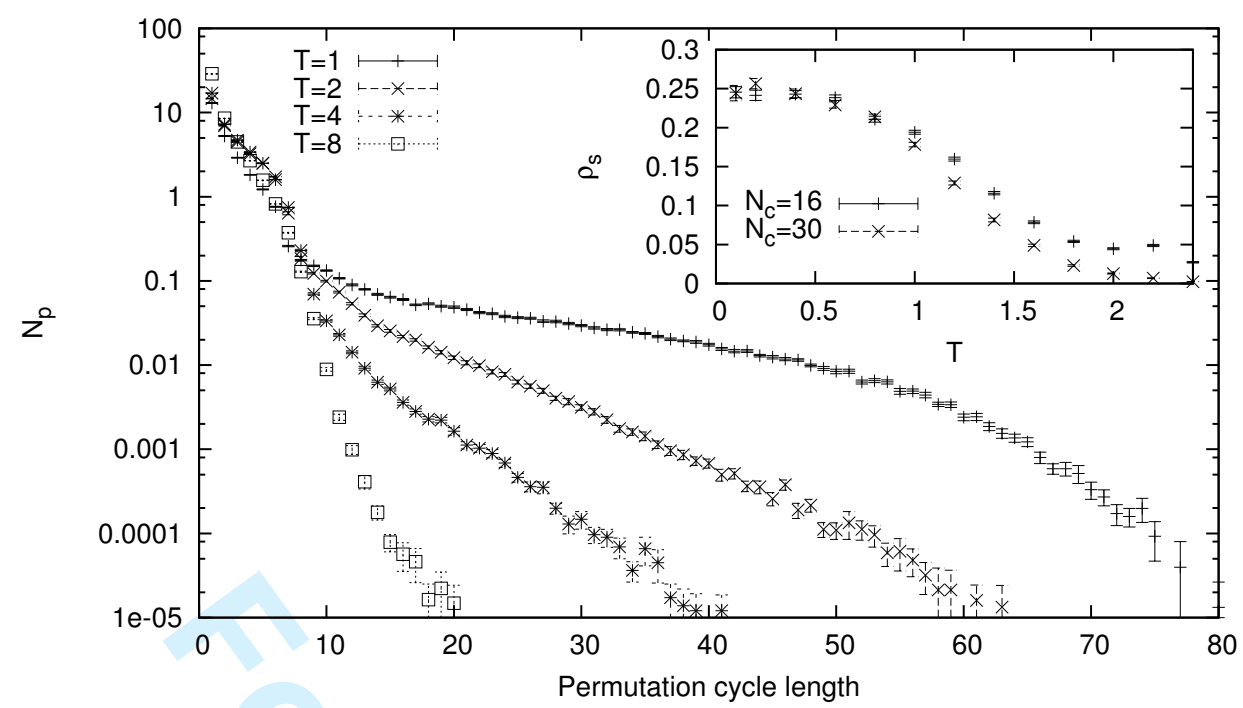

Figure 3. Average number of permutation cycles as a function of the permutation length for various temperatures (main panel), for $D=5, \mu=45, r_{s}=0.522$. The average number of particles per droplet is between 6 and 7 in these simulations, therefore the number of permutation cycles longer than this value drops at all temperatures. However at low $\mathrm{T}$ permutation cycles involving a large number of droplets become possible: this allows for dissipation-less flow in the system. In the inset the superfluid fraction $\rho_{s}$ as a function of the temperature is shown, for two different system sizes, containing $N_{c}=16$ or $N_{c}=30$ cristalline sites.

progressively impaired. On the other hand shorter permutation cycles still occur, indicating that individual clusters remain superfluid even at temperatures where global phase coherence is lost. Long permutation cycles have a direct bearing on the superfluid fraction, shown in the inset of Figure 3 a function of the temperature for various system sizes. Data are consistent with a superfluid transition in the Kosterlitz-Thouless universality class, [11] as expected for a two-dimensional system.

The finite superfluid response is accompanied by a strong signature in the momentum distribution $n(k)$, shown in Figure 4. Even though Bose condensation is suppressed in two dimensions at finite temperature, in the supersolid phase the calculated momentum distribution features a pronounced peak at $k=0$, as well as a smaller peak at $k=2 \pi / l$. Neither is found in the insulating crystal. The former represents the integral of the occupation number of low-momentum states, corresponding to a slow power-low decay of the one-body density matrix (OBDM). The latter describes a substantial real-space modulation of the OBDM with the periodicity of the triangular lattice. While modulations in the OBDM are expected also in the insulating case [12], they are too small to be detected within the statistical precision of this calculation.

It is interesting to consider the limit on the superfluid fraction induced by density modulations, as pointed out by Leggett [13]. The bound reads as [14]:

$$
\begin{gathered}
\rho_{s} \leq \min _{\varphi(\mathbf{r})}\left(\frac{1}{\rho V v_{0}^{2}} \int \rho(\mathbf{r})\left|\mathbf{v}_{s}(\mathbf{r})\right|^{2} \mathrm{~d} x\right) \\
\mathbf{v}_{s}(\mathbf{r})=\left(\frac{\hbar}{m}\right) \nabla \varphi(\mathbf{r}) \\
\varphi\left(\mathbf{r}+\left(n L_{x}, n^{\prime} L_{y}\right)\right)=\varphi(\mathbf{r})+2 \pi n^{\prime \prime}
\end{gathered}
$$

where $\varphi(\mathbf{r})$ is a phase function, $\rho$ and $v_{0}$ are the average value over the simulation cell of $\rho(\mathbf{r})$ and $\mathbf{v}_{s}(\mathbf{r})$ respectively, and $n, n^{\prime}$ and $n^{\prime \prime}$ are integers. This essentially 
Figure 4. Momentum distribution $n(k)$ for $D=5, r_{s}=0.522, \mu=45$ in the supersolid phase $(T=0.2$, red disks) and in the insulating cluster solid ( $T=4$, blue circles). The peaks at $k=0$ and $k=1$ (in units of $2 \pi / l$ ) are due to (quasi)condensation and strong modulation of the OBDM with the lattice periodicity, respectively.

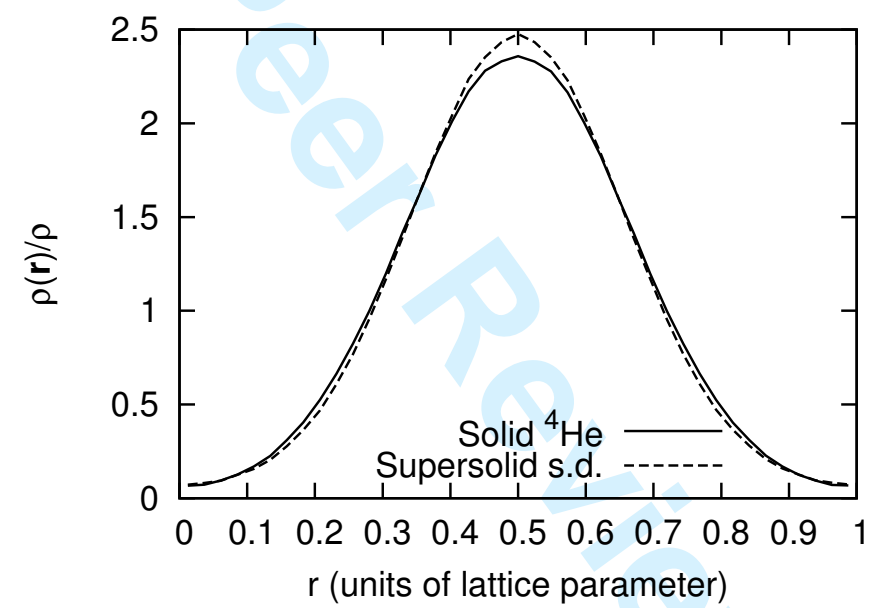

Figure 5. Similarity of the density profiles $\rho(\mathbf{r}) / \rho$ for soft disks in the supersolid phase $(\mathrm{D}=5, \mathrm{~T}=0.1$, $\left.\mu=45, \rho_{s}=0.25 \pm 0.01\right)$, and for solid ${ }^{4} \mathrm{He}$ near melting $\left(\rho=0.0765 \AA^{-1}, T=1 \mathrm{~K}\right)$. These density profiles are obtained along the crystallographic direction $[1,0]$ passing trough a lattice site. $r$ is in units of the lattice parameter.

states that if the system is uniform the superfluid fraction can be one, while if density modulations are present its value must be necessarily lower. Although the Leggett bound is very loose in strongly correlated systems such as solid ${ }^{4} \mathrm{He}$ or parahydrogen [14], it could provide an argument to explain why soft disks are supersolid under appropriate conditions while two-dimensional solid ${ }^{4} \mathrm{He}$ is never, should their density profiles look different. In order to verify this possibility we compared (Figure 5) the density profile of the supersolid soft disk system for $\mathrm{D}=5$, $\mathrm{T}=0.1, \mu=45, \rho_{s}=0.25 \pm 0.01$ with the one of solid ${ }^{4} \mathrm{He}$ in two dimensions at low temperature slightly above the melting density $\left(\rho=0.0765 \AA^{-1}, T=1 K\right)$. Actually the two density profiles are remarkably similar, and the Leggett bound gives $\rho_{s} \leq 0.39$ for the soft disk and $\rho_{s} \leq 0.37$ for Helium. This shows that, within the constraint of Eq. 3 imposed by one-body properties, many-body effects have even qualitative effects on the superfluid response. In particular, the difference between hard- and soft-core systems is entirely encoded in two-body (and possibly 


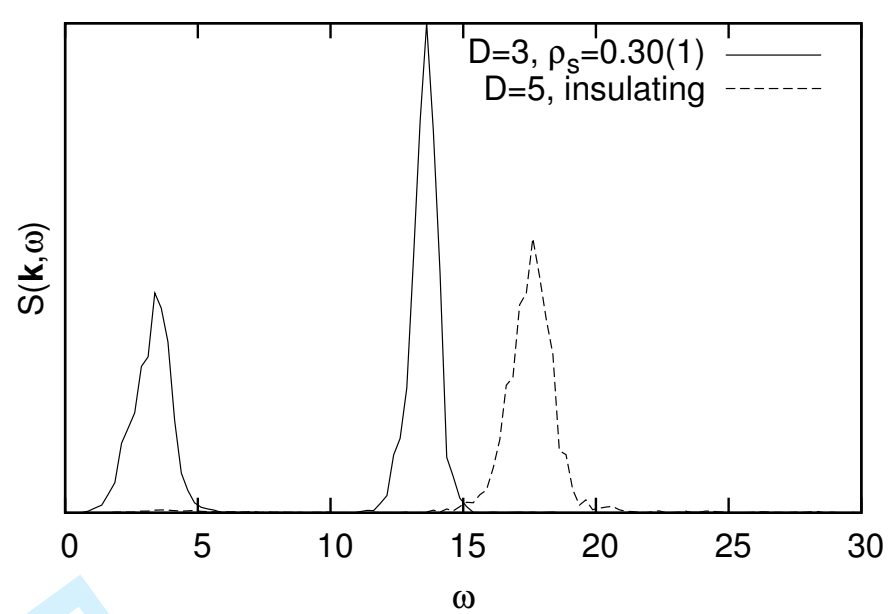

Figure 6. $S(\mathbf{k}, \omega)$ obtained with the described inversion procedure, for $\mathbf{k}=(0.25,0)$ in units of reciprocal lattice spacing. Data are obtained for two values of $\mathrm{D}=3$ and $\mathrm{D}=5$ at $r_{s}=0.421$, for $T=0.5$. In the former condition the system is supersolid, in the latter is a normal cluster solid.

higher) correlations. Indeed the pair distribution function of supersolid soft disks is peaked at contact [7], as opposed to the correlation hole induced by hard-core repulsion in Helium.

We finally discuss the dynamic structure factor $S(\mathbf{k}, \omega)$ of the system, which is related to the elementary excitations such as phonons in the solid and rotons in the superfluid. It is not obvious in advance if the supersolid has a dominant mode of mixed character or distinct branches. The worm algoritm is a path integral Monte Carlo approach, and therefore one cannot have access to real time dynamics of the system. Instead the imaginary-time density-density correlation function can be computed

$$
F(\mathbf{k}, t)=\frac{\operatorname{Tr}\left(\rho_{-\mathbf{k}} e^{-t H} \rho_{\mathbf{k}} e^{-(\beta-t) H}\right)}{N Z} \quad \rho_{\mathbf{k}}=\sum_{j<N} e^{i \mathbf{k} \cdot \mathbf{r}_{j}}
$$

$Z$ being the trace over the density matrix of the system. The $F(\mathbf{k}, t)$ is related to $S(\mathbf{k}, \omega)$ by an inverse Laplace transform:

$$
F(\mathbf{k}, t)=\int \mathrm{d} \omega e^{-t \omega} S(\mathbf{k}, \omega)
$$

The numerical inversion of a Laplace transform is a classical ill-conditioned problem. To cope with this problem, various approaches have been developed, among which the Maximum Entropy (ME) method is probably the most used[15]. Here we resort instead to the Genetic Inversion via Falsification of Theories (GIFT)[16] approach, which offers a better resolution in the reconstructed spectra. For example, when applied to superfluid ${ }^{4} \mathrm{He}$, GIFT is able to correctly separate the sharp quasi-particle excitations from the multiphonon branch, with appropriate spectral weights[16], whereas ME merges both structures[17, 18].

A typical result of the GIFT inversion procedure is reported in Figure 6, for $\mathbf{k}=(0.25,0)$ in units of reciprocal lattice spacing and for two different interaction stengths $D$ at the same density $r_{s}=0.421$ and temperature $T=0.5$. For $D=5$ (solid line) the system is in the insulating cluster solid phase, and the spectral weight is concentrated in a single phononic peak. For $D=3$ the system is in the supersolid phase, with a significant superfluid fraction $\left(\rho_{s}=0.30 \pm 0.01\right)$. The phononic peak is seen to shift to lower energies with respect to the insulating 
phase because the system is softer (see Figure 2). More interestingly, the spectrum displays a second peak with lower energy. Preliminary data on the dispersion of these spectral features suggests that both modes are acoustic. We thus conclude that a supersolid can sustain two distinct long-wavelength excitations, respectively related to the lattice dynamics and to the superfluid response.

\section{References}

[1]E. Kim and M. H. W. Chan, Nature, 427, 225 (2004); Science 305, 1941 (2004)

[2] See, for instance, S. Balibar, Physics 3, 39 (2010).

[3]F. Cinti, P. Jain, M. Boninsegni, A. Micheli, P. Zoller and G. Pupillo, Phys. Rev. Lett. 105, 135301 (2010).

[4] M. Lukin et al., Phys. Rev. Lett. 87, 037901 (2001).

[5]B. M. Mladek, P. Charbonneau, C. N. Likos, D. Frenkel and G. Kahl, J. Phys.:CM 20, 494245 (2008).

[6]C. N. Likos, A. Lang, M. Watzlawek and H. Löwen, Phys. Rev. E 63, 031206 (2001).

[7]S. Saccani, S. Moroni ,M. Boninsegni, Phys. Rev. B 83, 092506 (2011).

[8] M. Boninsegni, N. Prokof'ev, and B. Svistunov, Phys. Rev. Lett. 96, 070601 (2006).

[9] M. Boninsegni, N. Prokof'ev, and B. Svistunov, Phys. Rev. E 74, 036701 (2006).

[10]E. L. Pollock and D. M. Ceperley, Phys. Rev. B 36, 8343 (1987).

[11] J. M. Kosterlitz and D. J. Thouless, Prog. Low Temp. Phys. 7, 371 (1978).

[12] E. Vitali, M. Rossi, F. Tramonto, D. E. Galli, and L. Reatto, Phys. Rev. B 77, 180505 (2008)

[13] A Leggett, Phys. Rev. Lett. 25, 1543 (1970).

14]D. E. Galli, L. Reatto, W. M. Saslow, Phys. Rev. B 76, 052503 (2007).

[15]J. E. Gubernatis and M. Jarrell, Phys. Rep. 269, 135 (1996).

[16] E. Vitali, M. Rossi, L. Reatto, and D. E. Galli, Phys. Rev. B 82, 174510 (2010).

[17] M. Boninsegni and D. M. Ceperley, J. Low Temp. Phys. 104, 339 (1996).

[18]S. Baroni and S. Moroni, Phys. Rev. Lett. 82, 4745 (1999). 